\title{
CRISPR sur embryons \\ humains : une nouvelle \\ étape
}

Bertrand Jordan

L'actualité récente au sujet de la technique CRISPRCas9 a été dominée par le démarrage des premiers essais cliniques de thérapie génique en cancérologie [1] $(\rightarrow)$ ainsi que par une première décision dans la querelle des brevets sur cette méthode [2], querelle qui, vues $\rightarrow$ Voir la Chronique génomique de $B$. Jordan, $\mathrm{m} / \mathrm{s} \mathrm{n}^{\circ} 11$, novembre 2016, p. 1035 les sommes en jeu, va sans nul doute connaître de multiples rebondissements. Le thème de la modification d'embryons, amorce d'une thérapie génique germinale, connaissait par contre une certaine accalmie après la publication de deux articles rapportant des tentatives pratiquées sur des embryons non viables [3, 4] et qui montraient les limites actuelles de la technologie. Mais un nouvel article [5] récemment paru relance la question: il rapporte en effet la correction de mutations dans des embryons viables, donc pouvant en principe être implantés. De plus ces embryons ont été spécialement produits dans le but d'être utilisés pour cette tentative, en infraction aux règles mises en place dans de nombreuses nations - mais pas en Chine. Cette nouvelle publication, qui n'a pas connu un aussi large écho que les précédentes, mérite cependant d'être examinée tant du point de vue de ses résultats que des questions qu'elle soulève.

L'article en question, mis en ligne le $1^{\text {er }}$ mars 2017, paraît dans une revue de bon niveau (Molecular Genetics and Genomics [MGG], facteur d'impact 2,6). Intitulé CRISPR/Cas9-mediated gene editing in human zygotes using Cas9 protein [5], il implique cinq équipes chinoises ainsi qu'un chercheur (chinois) du Houston Fertility Institute, Texas, États-Unis. L'institution principale est le centre de médecine reproductive de l'université médicale de Guanzhou (Canton). Dans les grandes lignes, il s'agit d'une série d'expériences sur des embryons non viables (comportant trois pronucléus, dits «3PN») qui a servi de mise au point préalable, suivie de la production d'embryons viables («2PN », comportant le nombre normal de pronucléus) portant une mutation et de la correction de cette
UMR 7268 ADÉS, Aix-Marseille, Université/EFS/CNRS, Espace éthique méditerranéen, hôpital d'adultes la Timone, 264, rue Saint-Pierre, 13385 Marseille Cedex 05, France ; CoReBio PACA, case 901 , parc scientifique de Luminy, 13288 Marseille Cedex 09 , France.

bertrand.jordan@univ-amu.fr brjordan@orange.fr

mutation. Sur le plan

technique, ces chercheurs procèdent par injection dans l'embryon de la protéine Cas9 complexée avec I'ARN guide (ARNg), plus éventuellement l'oligonucléotide «donneur» destiné à la réparation de la mutation. Les embryons 3PN proviennent du tri effectué lors des fécondations in vitro (ils sont habituellement éliminés) ; dans un premier temps on teste l'introduction de délétions correspondant au mécanisme NHEJ (nonhomologous end joining), puis la modification de la séquence d'un gène $(G 6 P D \text { ou } H B B)^{1}$ en ajoutant un $A D N$ donneur et en veillant à ce que l'embryon soit en phase $S$, plus favorable au mécanisme de réparation homologue (HDR, homology-directed repair). Ces expériences préliminaires montrent une bonne efficacité, environ $60 \%$, pour l'introduction de délétions ciblées dans un gène via le mécanisme NHEJ, le plus efficace, et un rendement plus faible, 10 à $20 \%$, pour l'introduction de modifications précises dans un gène via le mécanisme HDR. Dans l'ensemble, ces expériences, qui sont présentées avec les témoins adéquats, donnent des résultats cohérents avec ceux des publications précédentes sur les embryons 3PN [3, 4], avec une efficacité un peu supérieure que l'on peut sans doute attribuer à l'injection de Cas9 sous forme de protéine complexée avec son ARNg (et non sous forme d'ARN messager devant être traduit dans le cytoplasme de l'embryon).

${ }^{1}$ G6PD code la glucose-6-phosphate déshydrogénase ; HBB code la bêta-globine. 
Pour l'étape suivante, qui est en quelque sorte une démonstration de thérapie génique germinale, il faut disposer d'embryons portant la mutation à corriger: les auteurs vont donc produire de tels embryons. Ils utilisent pour cela des ovocytes normaux (wildtype, écrivent les auteurs) mais immatures, peu adaptés à une fécondation in vitro normale et donc aisément disponibles, qu'ils soumettent à une étape de maturation in vitro. Ils sont ensuite fécondés par injection intracytoplasmique (procédure ICSI, intra cytoplasmic sperm injection) avec des spermatozoïdes provenant de donneurs présentant soit une thalassémie (mutation dans le gène $H B B$ à l'état hétérozygote), soit une affection métabolique appelée favisme (mutation inactivant le gène G6PD situé sur le chromosome $\mathrm{X}$ ). Ils obtiennent dans chaque cas 10 embryons, dont le génotype précis est inconnu puisqu'on ne sait pas quel spermatozoïde les a fécondés, et procèdent sur chacun d'eux à l'injection du complexe Cas9-ARNg plus un oligonucléotide donneur (90 nucléotides) pour corriger la mutation si elle est présente. Après deux jours de culture, les embryons sont lysés pour analyse de leur ADN (la question de leur implantation ne se pose donc pas). C'est l'étude de cet ADN, provenant de la dizaine de cellules qui constituent l'embryon à ce stade, qui va indiquer si ce dernier portait ou non la mutation à corriger, et si la correction a bien eu lieu : I'ADN donneur pour HBB ou pour G6PD porte une substitution silencieuse qui fait apparaître un site de restriction en cas de réparation HDR et permet de distinguer l'allèle «sauvage » de l'allèle mutant et corrigé. Par exemple, pour la correction de G6PD, huit embryons sur 10 portent des séquences du chromosome $Y$ : ils sont « $X Y$ », ont été fécondés par un spermatozoïde contenant le chromosome $Y$ et n'ont donc pas reçu le gène G6PD mutant. L'analyse de l'ADN des deux autres embryons, qui sont « $X X »$, montre pour l'un la présence en quantité équivalente d'un allèle sauvage (provenant de l'ovocyte) et d'un allèle corrigé (mutant provenant du spermatozoïde et correctement rectifié par HDR) : c'est le résultat idéal attendu. L'autre embryon $X X$ contient trois allèles: sauvage, corrigé, et portant une délétion de quatre nucléotides, dans un rapport de $2: 1: 1$. Il est interprété comme une mosaïque, la moitié des cellules contenant un allèle sauvage et un allèle corrigé, l'autre un allèle sauvage et un allèle modifié par NHEJ. Pour le gène $H B B$, quatre embryons sur dix portaient la mutation et ont été édités, dont deux par recombinaison homologue - mais avec là encore la présence de mosaïques. Les recherches de modifications parasites (hors cible) n'ont pas été systématiques, mais le séquençage (à la redondance 30x) de l'ADN de l'unique embryon G6PD correctement corrigé n'a pas montré de tels évènements. Au total, cet article montre bien la rectification d'une mutation ponctuelle par action de Cas9 sur des embryons viables mais cette réparation n'a été réellement effective que dans un cas sur six. C'est évidemment trop peu pour envisager une mise en œuvre clinique, mais cela n'en représente pas moins une étape importante. Notons aussi que les embryons viables semblent mieux se comporter que les embryons 3PN, et que l'emploi de la protéine Cas9 (commercialement disponible) plutôt que son ARN messager est à recommander.

Comme je l'indiquais plus haut, cet article semble être passé presque inaperçu lors de sa publication : bien qu'il ait été mis en ligne le $1^{\text {er }}$ mars 2017, ni Nature ni Science ne s'en sont fait l'écho immédiatement. II marque pourtant une étape notable vers la faisabilité technique de la thérapie génique germinale, et soulève également de sérieuses questions éthiques. D’ailleurs la revue qui le publie a pris la précaution de faire paraître le même jour un «commentaire éditorial » [6] qui résume l'article, rappelle ses limites, et explique qu'il a été longuement analysé à travers trois versions successives avant publication - il avait été soumis à l'origine début juillet 2016. Ce commentaire mentionne aussi que ces recherches ne seraient pas autorisées dans de nombreux pays, et termine en affirmant que la revue ne prend pas position dans les discussions sur l'emploi de cette technique appliquée aux embryons humains. Selon des rumeurs difficiles à vérifier, plusieurs travaux similaires auraient été réalisés mais se heurteraient à de grandes difficultés pour être publiés. Pour revenir à l'article de Tang et al [5] on peut noter que ces recherches ont été approuvées par un comité d'éthique, mais que - comme pour les précédents articles chinois sur le même sujet $[3,4]$ - il s'agit uniquement du comité local de l'hôpital dans le cadre duquel sont réalisés les travaux. II n'est pas certain qu'une telle structure jouisse de toute l'indépendance requise pour statuer sur un sujet délicat touchant au prestige de l'institution. Le point le plus litigieux concerne la production d'embryons dans un but de recherche: elle est interdite en France ${ }^{2}$, comme dans la plupart des nations européennes et - de manière moins nette car dépendant des États - aux États-Unis. Ce n'est effectivement pas le cas en Chine, où il suffit que les cellules germinales aient été librement données par des volontaires...

Ce travail confirme en tous cas la place que prend la Chine dans les applications cliniques de la technique CRISPR-Cas9. Premiers essais sur des embryons humains (non viables) [3], premier essai de thérapie génique [1] $(\rightarrow)$, et maintenant premier essai sur des embryons viables [5]. Faut-il $\rightarrow$ Voir la Chronique génomique de $B$. Jordan, $\mathrm{m} / \mathrm{s} \mathrm{n}^{\circ} 11$, novembre 2016, p. 1035 s'attendre à la première implantation d'embryons génétiquement «réparés » ou modifiés? II reste de sérieux obstacles techniques avant d'en arriver là. Compte tenu du faible taux de succès, qui ne va sans doute pas avant longtemps s'approcher du $100 \%$ nécessaire pour pratiquer de telles implantations à l'aveugle, il restera indispensable de vérifier que la modification souhaitée a bien eu lieu dans toutes les cellules de l'embryon. II est concevable d'analyser une cellule de ce dernier avant l'implantation, et de vérifier ainsi le bon déroulement

\footnotetext{
${ }^{2}$ Notre loi stipule: « Une recherche ne peut être menée qu'à partir d'embryons conçus in vitro dans le cadre d'une assistance médicale à la procréation et qui ne font plus l'objet d'un projet parental. »
} 
de l'opération; mais comment s'assurer que l'on n'est pas en présence d'une mosaïque sans analyser chaque cellule et par la même détruire l'embryon? Cela semble impossible, et il faudra sans doute changer de stratégie. Il est concevable que l'on puisse procéder à la modification du génome sur des cellules souches pluripotentes induites (IPSC, induced pluripotent stem cells) dérivées de cellules somatiques, puis après sélection des cellules correctement modifiées, les conduire à se différencier en cellules germinales. L'essentiel des étapes a déjà été réalisé chez la souris, il n'est pas inimaginable de le faire chez l'homme - mais les inconnues et les problèmes sont nombreux. En tous cas cet article nous rappelle une fois de plus que les possibilités de modifications génétiques sur embryons humains continuent à progresser, et que des chercheurs - notamment en Chine - n'hésitent pas à en explorer les applications même lorsqu'elles impliquent des interventions qui ne font pas l'objet d'un consensus international. $\diamond$

\section{SUMMARY}

A new milestone in human embryo editing

Viable human embryos carrying a disease mutation have now been corrected by CRISPR-Cas9 editing. Although the correction is not perfect, and the embryos have not been implanted, this brings us closer to human germline editing and to the associated ethical questions. $\diamond$

\section{LIENS D'INTÉRÊT}

L'auteur déclare n'avoir aucun lien d'intérêt concernant les données publiées dans cet article.

\section{RÉFÉRENCES}

1. Jordan B. Les débuts de CRISPR en thérapie génique. Med Sci (Paris) 2016 ; 32 : 1035-7.

2. Ledford H. Broad Institute wins bitter battle over CRISPR patents. Nature 2017 ; $542: 401$

3. Liang P, Xu Y, Zhang X, et al. CRISPR/Cas9-mediated gene editing in human tripronuclear zygotes. Protein Cell $2015 ; 6: 363-72$.

4. Kang $X$, He W, Huang, $Y$, et al. Introducing precise genetic modifications into human 3PN embryos by CRISPR/Cas-mediated genome editing. J Assist Reprod Genet 2016 ; $33: 581-8$.

5. Tang L, Zeng Y, Du H, et al. CRISPR/Cas9-mediated gene editing in human zygotes using Cas9 protein. Mol Genet Genomics 2017 Mar 1. doi: 10.1007/s00438-0171299-z.

6. Hohmann S. Editor's comment on "CRISPR/Cas9-mediated gene editing in human zygotes using Cas9 protein". Mol Genet Genomics. 2017 Mar 1. doi: 10.1007/ s00438-017-1300-x.
TIRÉS À PART

B. Jordan

P ossédées du malin au Moyen-Âge, les sorcières hystériques sont vouées au bûcher. Enfermées au XvII siècle, maltraitées, elles rejoignent la Cour des Miracles de l'Hospice de la Vieillesse-Femmes à la Salpêtrière... Jusqu'à ce que le Dr JeanMartin Charcot (1825-1893) mène le combat qui transforme l'ancien hospice en hôpital : l'École de la Salpêtrière de

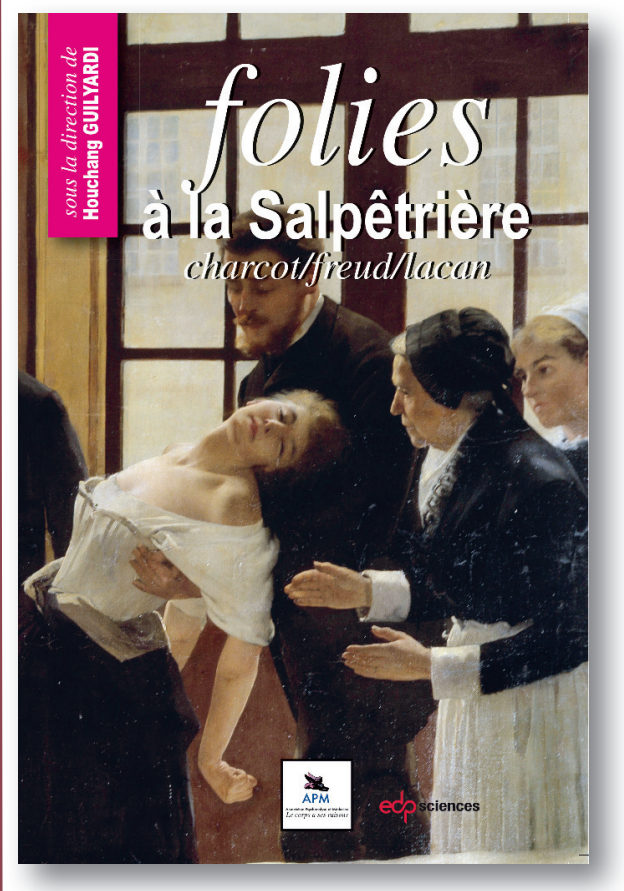

ISBN : $978-2-7598-1268-4$
240 pages Paris est née, qui devient lieu de recherche, d'enseignement et de soins, de renommée internationale.

Jean Martin Charcot n'a pas bonne presse, et pourtant... Hystérie et folie traversent les siècles, prenant les formes de «l'air du temps ».

De l'utérus migrateur d'Hippocrate aux recherches neurologiques de Charcot. Du désir inconscient avec Freud à la jouissance du parlêtre chez Lacan... C'est à cette traversée historique et conceptuelle que nous convie cet ouvrage.

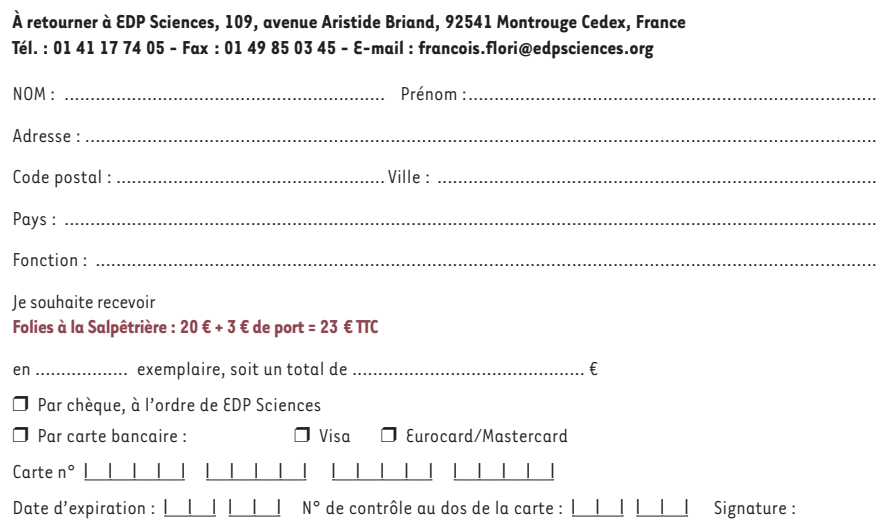

À retourner à EDP Sciences, 109, avenue Aristide Briand, 92541 Montrouge Cedex, France

Code postal : .

Je souhaite recevoir

$\square$ Par chèque, à l'ordre de EDP Sciences

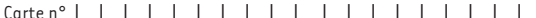

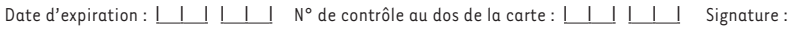

